\title{
Consciousness: A Paradigm Towards Artificial General Intelligence
}

\author{
Adnan Rashid Chaudhry
}

\begin{abstract}
Consciousness nearly in all its elusive history is a convoluted notion, often misconstrued or not understood enough to cause a reproducible representation. With these odious assertions, this publication is opening the box of consciousness with deviation from commonly understood notion of consciousness. The proposed paradigm of consciousness approaches this issue with speculative and intuitive perspectives, essentially it is a precursor activity in hope to materialize the elusive artificial general intelligence, the true carrier of exceptional human intelligence and consciousness. This paper posits a counterbalance approach to the current paradigm of consciousness and as an alternate a radical theory of consciousness is presented. This attempt on the behavioral, structural and functional working of consciousness is kept pragmatic in the intractable universe of consciousness.
\end{abstract}

Index Terms-Consciousness, Existence, Mind's Model of the World, Network of beings, Artificial general intelligence, Data driven approaches.

\section{INTRODUCTION}

Consciousness is both perplexing and elusive concept, and in common parlance, it has far too many interpretations, meanings and references. All of them, describe some aspect of mental states, if that complexity was not enough, there is a framework of self referencing, and any of the semantic representation of consciousness depends only on the perspective of who uses it, what is the context and in which peculiar knowledge domain? In all of this misty convoluted descriptions associated with the phenomena of consciousness, the commonly understood terms identified with it are thinking, awareness, feeling, experiencing, metaconsciousness, learning, recalling, memorising, intentions and understanding etc...

Scanning through history of consciousness in human beings and beyond, we are faced with various approaches, perspective and interpretations to the question of consciousness, viz. monoism (Alter and Pereboom (2019)), dualism (Descartes (2003)), materialism (Papineau (2002)), physicalist, emergentism (Haugeland (2014)), panpsychism (Chalmers (2015)), society of mind (Minsky (1988)), connectionist (Lloyd (1995)), frame theory (Blackledge (2003)), many draft theory (Dennett (1993)) and rule based (Weiss and Indurkhya (1995)) and language based (Searle et al. (2002)). All of them in one way or the other have a treatise on the issue of consciousness. The main type of inquiries these theories try to answer are transcendental role of consciousness, the grounds and basis of its existence, its manifestations from quantum to human levels, and among other one futuristic issue is how to replicate it in machines, generally known as Artificial General Intelligence (AGI). These questions are intriguing and perplexing, and the convoluted nature of interpretations on the subject of

- Mr. Adnan Rashid Chaudhry is with the Department of Computer Science, Faculty of Computing and Information Technology, Northern Border University, Rafha, KSA.

E-mail: adnan.chaudhry@nbu.edu.sa, adnan3413@gmail.com ORCID: 0000-0002-5137-9108 consciousness only adds to complexity and intractability.

In any study on the subject of consciousness, these assertions on the issue of consciousness get veritably muddled by the term consciousness and in this ambiguity, this publication is an attempt to augment the assertion space to form a theory of consciousness that is simple, intuitive, clear and pragmatic to the enigma created by above mentioned issues. Any pragmatic approach that may address these issues of consciousness is important to human civilization and its development, but it may have far reaching consequence to life as we know and spawn a future of a transcendental evolution of sentience beings. It is important to note that there is no claims that this publication in any definite terms settle few of the above mention contentions rather may provide a newer perspective to the intractable nature of consciousness.

This publication subsumes various perspectives \& presents a general notion of consciousness in form of a model of world as a network of beings, and in this way, it firmly sets a divergent interpretations on consciousness. All of these concepts of consciousness though are personal to author, one principle aim is to disseminate these interpretations to others interested to develop, modify and extend consciousness in a pragmatic way to the benefits of humanity. The specific agreement to any of these divergent perspectives is not necessitated by the author rather if these concepts are entertained then the principle aim of the article is consummated. Secondary aim of the publication is to set premises for emergence of a new and applicable frameworks of AGI that may assist to progress the general aim of imbuing machine with intelligence and consciousness. This is a noble endeavor to spread consciousness as a structure, beyond this blue planet in the vastness of hostile universe. This aim is a mean of preservation of essence of life, by considering consciousness as a new DNA that has the potential to seed life and structures to the edges of reality. 


\subsection{Existence: Central Notion of New Paradigm}

Existence in this discourse has a general classification and it encompasses everything in realm of experience: physical or mental or even the speculative ones e.g. unicorns.

Among these existences, physical is too direct an experience to cast any aspersions and direct empirical instruments of validation are commonly applicable. Whilst mental is also intuitively accepted as common consensus of experience exist, although mental existence is more a subjective issue compare to physical and remains contentious as a type of existence. As we are familiar that there exists mechanisms for concept verification, in contrast to both physical and mental; the speculative existence type has a lesser association with verifiable possibilities, and it is unique among three existences by providing a freer canvas for generating new configurations and ideas.

Extending the discourse on consciousness to the sources of human understanding and knowledge is the first step towards a pragmatic deconstruction of consciousness. In this regards among the three kinds of existences, it is the speculative one as a property of mind that engenders major divergence in consciousness, effecting new and unheard hypothesizes based on a set of new principle assumptions. Among them only those ideas are accepted as verified facts that are supported by an empirical evidence and observations. The only verifiable mechanism acceptable to test the validity of claims and hypotheses are by using the instruments of scientific method. In modern human history, the classification of valid and accepted ideas are proven for consistency by rigorous verification and in contrast groundless hypotheses, that are harder to reproduce or observe are abandoned.

A pragmatic theory of consciousness that may assist in the developing an AGI ought to address the concepts of knowledge creation and representation outside wetware in a hardware. To this end, identification of areas of experiences that help in knowledge creation becomes important. This proposal identifies three sources of knowledge that are physical, mental and speculative, and these three notions encompass the modes of existence, and any reference in this discourse to existence subsumes all three forms of existence.

\subsection{Ranks of Consciousness}

Following categorization of consciousness is preferred and referenced as ranks of consciousness in this publication.

1) Elementary or physical or material consciousness references to matter, energy and fields; and all its diverse forms and properties.

2) Biological consciousness found in living beings as a general category and it is focused on supporting biological functions; an intuitive survival driven and procreating mode.

3) Higher order consciousness refers to human consciousness by referencing the exceptional advanced functions compared to other life, which only have shades of those advance intelligence. There are host of functions that distinguishes higher order consciousness particularly it includes meta-

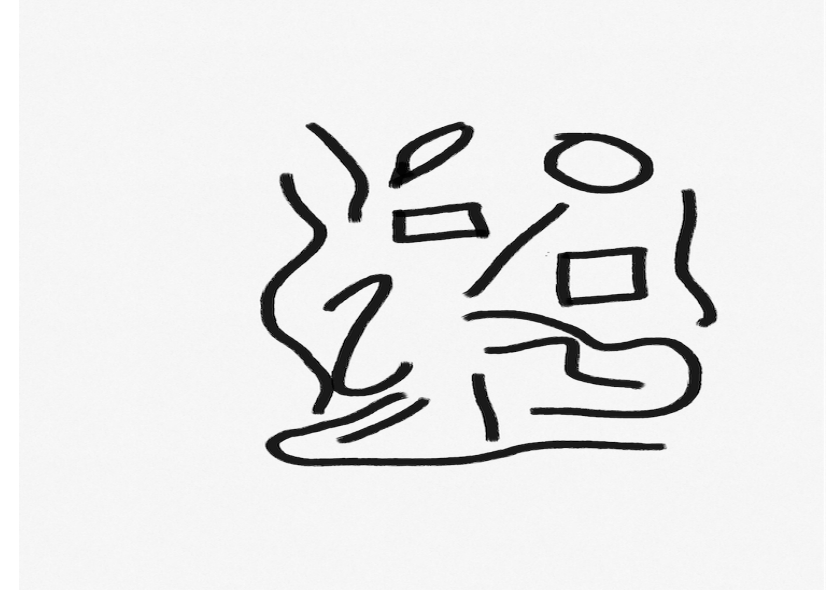

Fig. 1. Existences as come to being form a space of mutual consciousness, this is an entangled existence. Consciousness as a functional interpretation only arranges world to demonstrate semantic relations.

consciousness, thoughts, self-recognition, phenomenal experiences and so on.

\section{Model of Consciousness}

Awareness is the point of depart for an exploration in consciousness and in all things, awareness is product of sharing space, association and proximity of self, being or Heidiggers Desain (see further description in Heidegger (1988)) with aspects of reality. In layman term, one is aware of all forms and concepts that have physical and mental conceptions and if there is more to awareness then it is speculative and prognostics projections of mind driven plausibly from history of awareness (that may have sources in conscious or unconscious experiences).

\subsection{Theory of Consciousness}

The theory of consciousness that sets a suitable premise for AGI, preferably take a pragmatic paradigm and it ought to subsume the basic principles of clarity, generality and simplicity. In order to form such a theory, the intuitive answer to an inquiry in consciousness naturally starts at the interactive aspect of elements of nature. This interactive aspect accounts for all incipient modes of consciousness. This follows as a logical conclusion that all beings as such are consciousness by virtue of existence, this further entails that at simplest of scales, consciousness is manifested as an interaction of elements of nature e.g. collision and interaction of subatomic particle in sub-atomic structure. Second possible conclusion is that the augmentation of consciousness has functional diversity, beginning from physical to mental consciousness.

Physical consciousness is a manifestation of existence realizing as forms, behaviors and properties, and a mode of existence exerts stress in the physical system, and a noticeable prevalence of direct type of interactions aka a cause and an effect interactions. A memory based indirect or delayed responses are not necessarily remarked in such lower ranks of consciousness. While whenever alluding to mind, or mental, it embodies higher forms of consciousness, and a remarkable difference observable in this higher mode 
of consciousness subsumes delayed, processed and indirect responses based on a content of information as mean to relate with surroundings (In sentient beings it is thought). After all mental activity is an appropriate response dispensed in a way through biological extensions in biological beings. While moving further higher on the modes of consciousness, the ability to reflect is classified as one of the higher modes of consciousness along with functions such as abstract knowledge creation, planning and decision making etc... These higher order mental functions are employed and exercised by/in sentient beings to interact effectively in varying environments. One conclusion is that with developing consciousness beings begin to specialise for an existence, with varying capacities to either reconfigure themselves or environment for better adaptations.

Except for physical mode of consciousness that works basically on the principles of existence, from lower to higher modes of consciousness in beings can be understood as simple as "a sense and a response model" or the famous operant condition mechanism as response and stimulus by Skinner (1971). Although, at further higher orders the modes of consciousness veritably have more specialization of consciousness than this simple sense and response model. While, it is a good idea to apply a simple description on these disparate modes of consciousness in order to formulate an integrated theory, by focusing on peculiar similarities that joins them together, therefore in this perspective one suggestion is that consciousness has unity of existence and behaviors at all scales and ranks. The modes of consciousness are structural, functional and behavioral manifestations, differ only by sophistication.

\subsubsection{Space-Time \& Consciousness}

The proposed model of consciousness is developed in view of constructing an analogy with the model of space-time continuum (famously known in Physics proposed by Albert Einstein) and in space-time continuum, time has an entangled relation with space, in an analogous manners, consciousness has similar entangled relation with the spacetime continuum. To elaborate it further in this proposal, space-time model is explained taking time as an entity that does not represents a direct observable behavior, as a materialist will certainly consider as an existence. Time is rather an abstract concept emerging from connecting events in past, present and future by a sentient being or simply time can be explained as act of tracing the path of existences through space. The concept of time accounts for the events of existences.

In proposed framework, space is set of existences and a continued stream of presence of beings in this expanse of existences engender a concept of time, here, time is certainly considered as synchronous property of space. In the similar manner to time, consciousness demonstrates a higher order secondary property of existence by coexisting along side space-time continuum and this new continuum can also be understood as Space-Time-Consciousness. Space-TimeConsciousness encompasses all modes of interaction among the entities, which have an existence in a network of existences in time.
The following bullets detail caricatures of consciousness by considering the attributes similar to that of notion of time.

- If time play a role in the representation of causality of existence, so does consciousness plays, its similar role in making an other representation of causality describe in space-time continuum. Consciousness has its spectrum analogous to three concepts of time as past, present and future, but in form of interactions, and these three interactions are conjoined to describe the consciousness. An eventuality of three times gives the concept of memory, patterns and simply understanding as demonstrated in higher order of consciousness.

- Being in time is to assert cognizant of being in the presence of beings and helps to understand both time and consciousness in this network of existences.

- Mind with experiences is like a catalog and sometimes also referred to as a predicting machine: using past, developing future and acting out present.

This discourse on space-time- consciousness helps to understand the essentials of nature and behavior of reality in an integrated formulation.

\subsubsection{Universality of Properties}

To have a grasp of proposed model of consciousness, It is essential to comprehend the basis of reality assumed as a expanse of behaviors of existences. This assertion ensues that all properties from physical to mental are behavioral manifestations and any distinctions applied to them is one useful and second it is an act of human interpretation from anthropocentric perspective, (therefore criticism of pure reason is reasonable position by Kant (2002)). But eventually, consciousness is in essence representative of entities and relations among entities. Furthermore, consciousness in human is exercised through experiences of existences and thoughts engenders experiences and outcome is a system of communication. In regards to communication, language plays a pivotal role, and essentially, language enables a being to expresses in a structured from the consciousness by use of types and identities. Using this framework beings are able to make relations between behaviors of the reality. This mode of relating to reality engenders all sorts of useful distinctions, identities, patterns e.g. states of matter or another intuitive example is recognition of every object surrounding a being. While every distinction made to represent attributes of reality may in fact represent beings peculiar relations with the accessible expanse of behaviors of the reality, therefore there seems not a big harm to suggest that all properties are bounded in space-time continuum and all of them essentially are behavioral. Physical ones are more intuitive as physical objects are extraneous in existence, and physical behaviors are directly and empirically determined as objective treatment is possible. While mental representations are conjectured in minds to parlay the sophisticated experiences and are not determined except for their respective physical caricatures in the physical world.

Universality of properties as behavioral type is a fundamental premise of coining unity of all existences and enforces that all 
distinctions are in fact real and useful but based on functional and experiential types as contents of consciousness.

It is the unity of epistemology caused by consciousness, not the properties of nature themselves that derive the proposed universality of behaviors in this paradigm.

\subsubsection{Ontological Analyses}

Ontological analysis elucidates only universality of consciousness as this universe is ultimately an existence. if any distinctions in the notions of awareness and consciousness are consider, they are purely products of experiences of a being in network of existences. This paradigm of existence and consciousness shapes an intuitive interpretation suggesting that the diversity in existences engendering many modes of consciousness, likewise it entails the suggestion that when a form emerges, this act is simultaneous with an existence having two kinds of effects: physical and mental. Indeed, it is evidently reasonable to suggest that an identifiable structure is a being with extensions in physical or mental spaces. Every existence exhibits a distinctive and disparate form from the other forms, represented as a unique symbols in vocabulary of existences (symbols are treated in section [2.7]). Therefore, a form distinct itself from other forms, it exerts and claims an existence in reality. Coming to being is equal to consciousness, what how and why are intriguing processes of consciousness.

Consciousness stems out of the ontological commitment of a system, meaning existence of necessary ontology suggests that as soon as elements assumes a recognizable from by being at proximity then consciousness arrives synchronously to form the whole ontological space. In simple terms, an existence of things leads to consciousness of things, this further suggests that fundamental nature of consciousness as an existence in all modes and forms only emerges as modes of consciousness. Another aspect important in this paradigm of consciousness is that one is conscious of things in proximity although limited senses, perception and perspective of beings primarily restrict a categorical consciousness (this assertion is inspiration from critique of pure reason by Emanual Kant (2002)), and vice-a-versa, nonexistent beings will not synchronize to consciousness only an existence synchronizes to consciousness. Consequently, it is the objects that exist in ontology and made up the ontological commitments of a system and thus represent the complexity of consciousness. A corollary that follows from above discourse is the notion of non-existence of categorical consciousness.

\subsubsection{Time and consciousness}

The concept of time is a weighty figure in human experience, while on its own time does not present a form that is any way independently quantifiable in physical terms, rather it is existences that persists in space-time continuum and gives a secondary experience of time. A simple deduction that follows on the matter of nature of time is that time exist only to explain the existence. Existence evolves in time and space, and present a close intricate relation with both space and time. It appears like time is stitched with existence and in similar but less intuitive way consciousness is stitched with both existence and time. It is only the intuitive experience of natural order, observed in the ubiquitous manifestations of time that shapes its reality far better than the subjective nature of experience and consciousness.

Another property benevolent to the understanding of time in comparison to consciousness is general simplicity of notion of time. In general understanding time is more of an abstract concept that is closely associated to counting. This quantitative, intuitive and simpler treatment makes the notion of time more comprehensible to common sense and experience than utterly qualitative experience of mind and states presented in notion of consciousness. This inexplicable quality is rightly attributed as hard problem of consciousness by Chalmers (2007).

\subsubsection{Being-ness and Consciousness}

If existences engender consciousness then being-ness is also consciousness. Being-ness is quality of a being as it competes for space and existence by positively interacting with all elements around it. In the discussion of beings, an identification of a being is one of the fundamental issue of consciousness and primary motif of every form, as it comes to be in its embodiment. Beings entail an other important property of an impediment to change in their form, behavior and shape. Such properties like form, shape, behavior etc. are the contents of beings, and are preferably referred to as an assortment of essences of beings. All essences of a being eventuating to make it distinctive to everything around it in space-time continuum. Being exists then at some scale ought to be observable in space-time-consciousness continuum and the act of finding a being is consciousness.

\subsection{Synchronous Progress of Existence and Con- sciousness}

The proposed interpretation of consciousness makes it synonymous to existence, here it is not to portent that consciousness is an indiscriminable property of existence, like claimed by proponents of dualism in the mind-body problem (Descartes (2003)), rather this is an intuitive approach to consciousness that when being exist, they come to be in frame of consciousness of other beings. This interplay of elements at simpler scales is being and existence. From this, to elaborate the relationship between consciousness and existences, again consider the universality of properties, that asserts the suggestion of how all things in nature can be dispensed as behavioral/functional, and as functional/behavioral properties of beings come together then consciousness is an inevitable consequence. It follows that behavioral manifestations vary with diversity of beings and in case of physical entities, they are more rigid in behavior manifestations compared to biological ones. Furthermore, biological subsumes physical plus new behaviors and in this way a gradual progress of ranks of consciousness are seen from matter to life and finally culminating in homo-sapiens. In particular homo-sapiens, they manifest distinctions in consciousness by the unique essence in form of exceptional behaviors like: maneuverability, adaptation and innovativeness, which play an important role in their success as beings.

Understanding of progress of existences has a direct relation in understanding the progress of consciousness, as existences move from matter to life-to-sentient beings so does consciousness. Simpler causes are in physical consciousness and are easily understood as of its existence is 


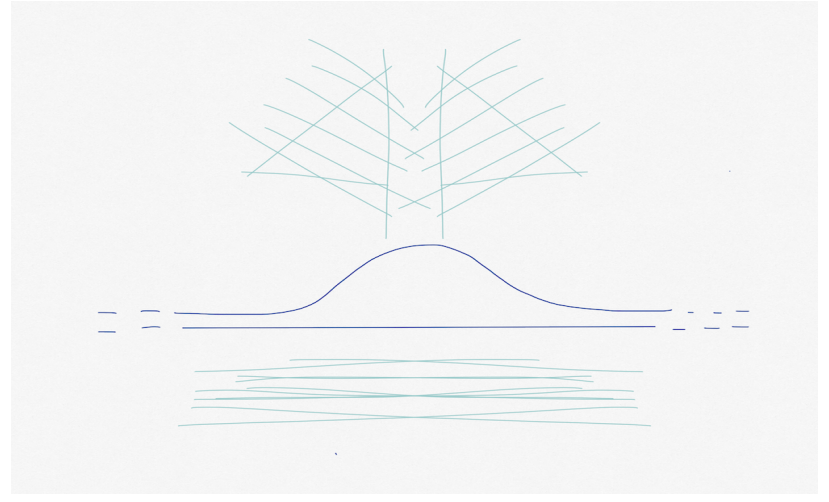

Fig. 2. Kernel relationship between consciousness ranks. Lower ranks are determined by horizontal lines while higher ranks are cross-pattern and in blue is a kernel relationship representation, stronger in the center and fades at peripheries.

physical in content. It follows that the responses of physical matter are in behavior of its properties, whereas sentient and animate beings are advanced forms of matter, and all of these animate beings have wider array of internal and external behaviors in determining the net expression and modes of consciousness. An evolutionary line of progression of consciousness is observable from very simple responses of behaviors of simpler existences to highly sophisticated responses of sentient beings.

In presented paradigm, in order to formulate consciousness as phenomena, we approach it differently from the usual assertion of creating consciousness/intelligence by strict mapping of inputs to outputs. The current practices in the intelligent systems designs rely on a large set of examples in order to approximate a behavior. Such approaches to intelligence and consciousness are narrowly focused on data samples and inadequate to generalise any problem, and only possible solution is by maintaining a stable less variable system, and inexorably new data diverging in behavior in any scale and dimension will have a negative effect on working of such an unconscious system (Beyer (2016)). Data samples suffer from incompleteness of representation and sample represents an essence of a function in an instance, not a function itself.

Contrarily, the proposed paradigm is realised as a codification of interactions and behaviors among elements of a world in a map and less focused on data samples. This formulation engenders the emergence of an integrated structural representation that may account for low to higher ranks consciousness, and the key aspect of this paradigm is the evolving functional or behavioral nature of learning integrated in the designs of intelligent systems.

Another important interpretation of presented paradigm of consciousness is the evolving conscious structures with a lax type of relationship among ranks of consciousness. Assume a lower consciousness ranks, it essentially works as a substrate for the higher ranks, in itself, this rank have behaviors that are independent and invariant to other ranks. Thus, among ranks not all variations in behaviors have quantifiable effects on the other layers and behaviors. A type of lax associations or links among these ranks of consciousness are assumed, a preferred analogy of relation between ranks is in shape of a kernel function Fig.2. The fundamental role of substrate ranks is to lay the foundations of new higher rank's structures that have a existence dependence on substrates, while these new higher conscious structures have unfathomable capacity to evolve, if and when the potential allows for structural explosion.

Consciousness in this paradigm is traceable in intelligent system design as in a behavioral/functional roles with integration of information that freely allows intelligence to evolve and to learn with a dynamic approach.

\subsubsection{Physical Elementary Consciousness}

Physical consciousness represents simplest form of existences in comparison to biological forms (as physical: essentially precedes biological), this insinuations alludes to quality of a physical being having a dual property of existence and consciousness. A being is only a being, if it demonstrates consciousness by being part of a network of associations, here in this network, consciousness is felt and experienced and originates from the interactions with proximal entities. Consider an example of a being, that is composite of atoms then it is an assortment of atoms that exhibits a self. A self exists, if and only if such a self has properties to preserve its form, behavior and existence.

It follows that "an ability of a being to preserve itself is a mode of consciousness."

\subsubsection{A Form}

In present paradigm of consciousness, the concept of reality is assumed as a composition of structures that are arranged in layers and scales, and the question of what constitute a form, being or discernible existence, becomes critical in building an understanding of consciousness and intelligence. A promising approach to describe a form is by appealing to intentionality (Ricoeur (1967)), an aboutness about existences. Following these premises a form in a materialist description is identified as an assortment of elements at a peculiar scale and having some identifiable properties. Another interesting description of form in context of our thesis is to consider a minimum set of essences that constitute a form. These concepts of form play a fundamental role in explicating the complexity innate in links and associations among forms, in a network of forms. Synchronously, an other important aspect to consider is the simplification of a form in network of forms. To come up with process of reification of the construct of forms is inexorable, if an imitational caricature of such forms are essential to realise that exist extraneous to the wetware like brain. The major obstacle is the identification of form relevant to a local network structure and it seems intractable without realising a self in a relevant scale. How to determine an interactive map of world based on inderterminable number of possible permutations of possible forms and links is an open question. This entails a necessity to construct a form, a basic conception that is more likely a relevant structure to the given scale and layer of reality.

A form is set of essences in a reality map and a form description is the associations it hold in a network of forms. This means forms semantics are not only a matter of internal structure rather it is also based on relations with aspects of reality, it exists at an instance of space-time-consciousness. 


\subsubsection{Phenomenology}

One of the major influence on the formulation of this proposal comes from phenomenology (Heidegger (1988)), which posits everything internal and external to the event of consciousness as sentient beings experience it. This philosophical tool lays an integrated and a complex structural analysis of consciousness, where phenomenological cognition resides and shares the same space as of higher ranks of consciousness presented in this publication. Where as the physical kind has roots in explaining beginning of consciousness and entails an existence as a principle subject matter of consciousness and one corollary of this premise of consciousness is an absence of consciousness in absolute emptiness, and where as we see a sharing of spaces by matter and mental forms with every form and element around them in building a structural edifice of consciousness. As the event of being creation is happening the idea of being perseverance is simultaneously acting out as necessitated by consciousness.

Phenomenology in any event describes and subsumes every elements that may influence higher ranks of consciousness as postulated in humans and it extends sound foundations for an epistemological inquiry into the structure of consciousness.

\subsubsection{Panpsychism}

Panpsychism appeals to the concept of soul to consciousness (see Chalmers (2015)), it is insinuated as an essential internal structure for the processing of information in every elements of nature from subatomic particles to human. Apparently, it is a similar insinuation to the concept of dualism but playing out at a larger scale. While in this proposal, simpler to higher ranks of consciousness make no such claim rather it appeals to existence and reification of being in existence necessarily embodying the aspect of consciousness. As the form assume more intricate and sophistication, lower rank consciousness of interaction reconfigures in augmented complexities to higher ranks of consciousness. The concept of protoconsciousness in realms of panpsychism, where elements and particle are assumed to carry a sense of encoded experience. In contrast the proposed physical consciousness is product of elements or particles sharing space-time continuum with others and in this soup of reality, every particle shares interactions and no special apparatus except from existence itself is necessitated to encode consciousness.

\subsubsection{Russellian Monoism}

Being in world as an existence has resemblance with monoism ( Alter and Pereboom (2019)) while in special case of Russellian monoism resemblance with present paradigm goes insofar as to only the primal constituents such as structuralism of physics, realism of quiddities and quiddities of consciousness. All three concepts of Russellian monoism are treated with conception of unity and no distinction are considered essential, not for internal/external groundedness, neither there is any relevance to rational and irrational treatise about behaviors and properties nor any insinuations to panpsychism or panprotopsychism of any kind. Strictly, in this proposal everything has universality and unity of existences and all distinctions are modes of behaviors, modes of existences and modes of consciousness. Some of these modes are proximal, that is to say near, so the interaction among such entities are considered as direct (such interactions of proximal being are a priori, given in the existence), like structuralism of physics. As the proximity and scale get modified, there is relevance to realism of quiddities, and more so, if by change of perspective or simply by protracted changes in scales, the form earlier identified may still exist at same scale and proximity, but now there is a lack of consciousness about the form at disparate scales and perspectives, its like accessibility is revoked and behaviors previously assume are out of consciousness, and thus there is a major role of change of intentionality.

The principle advantage of unity and universality of reality by behaviors is that it completely describe existences only by proximity of scale in space-time-consciousness.

\subsection{Supervenience}

In this discourse, there is major assumption on the subject of reality, and the preferred view is to consider reality as a hierarchical layered structure at various scales. Under these assumptions, the philosophical notion of materialism and supervenience are investigated for their prove of application to describe nature of consciousness. In this trial, even the strong empiricist are troubled as reality depicts presumably a structure upon structure configuration in layers and often, it is hard to verify the existence of a strong relation of type cause-and-effect among proximal layers, ergo these gaps in explanation are hindrance to a simple notion of relating and understanding reality and subsequently, it acts as a major obstacle in developing a pragmatic model of reality for an AGI system. For progressing the argument, we set aside materialist/empiricist approach of hard physical evidences of suggesting a determined mappings of structures/behaviors between layers, and instead, we opt for a more flexible notion of supervenience for the goal of understanding relation among structures in layers of reality. This change of approach like materialism do account for some behavior correspondence among structures in layers of reality but this piece-wise approach for few properties handled by materialism and then supervenience presents an inadequate representation to accommodate the varying nature of relationships among structures in layers of reality and in this regard a comprehensive approach is requisite to account for the behaviors in a more general and flexible manner.

Therefore in common observation, relationships of cause-effect type only seems to hold for a narrow range of behaviors among layers and for few properties supervenience assertion may hold and then a continuum of intractable properties, thus in this proposal, mapping is proposed to have a functional type to account for the changing nature of relations between structures and behaviors in layers of reality. One promising approach is the using the kernel function to accommodate changing relationship between structures and behaviors of layers of reality, to elaborate it has a narrow band of highly traceable relations between structures/behaviors, and these behaviors satisfies causeand-effect type mapping and then a weak relation persists for another set of behaviors and finally in rest of behaviors both layers have un-mapped behaviours. Furthermore, in 
kernel explanation of reality, well mapped properties are termed as primary type between layers but remaining behaviours/properties between layers are often hard to verify. There is one major assertion in regards to layered and scaled reality is that the underlying micro-structure must be laying the substrate for further existences and the vast set of behaviors among layers show no mapping, therefore evidently diverging from the supervenience (Kim (2017)) assertion of "A-properties differ then B-properties must differ".

A functional description of relationship between structures arrange in layers and scales of reality is a comprehensive approach then limited explanatory theories that are subscribed based on type of relation observed, such as strong causal or weak supervenience types in separate description of relating properties and behaviors among layers. To put things in perspective, each substrate at some scale lays down a framework for higher existences and it may demonstrate a stronger relationships in mappings of few properties, beyond these causal properties, the relationship diminishes and higher layers demonstrate independence in their multiplicity of forms, structures and behaviors. Here the proposal is that the engendered association is only stretched to lay the framework between layers and after that substrate/micro-structure plays no major role in functional or functional roles or forms found in higher layers. Substrate layer structures only support the essential existence with few traceable properties associating them to properties of higher layer structures, whereas forms and structures in a layer are causally determined by interaction with structures in proximity of their scales.

These assertions play a role in reshaping interpretations of reality and in our concern understanding of development and existence of consciousness, particularly the physical consciousness and the cognitive processes of sentient beings. They are two structures in two proximal layers, both of them share spaces and overall form of relationship could be adequately described by use of a kernel function. To summarise the kernel function between structures in layers of consciousness, there is a strictly causal area in middle of the kernel ( see Fig. 3) e.g. functional neurons and brain in a living being produce consciousness. The close proximity to the center area of the kernel function represents relation like supervenience e.g. neuro-science is in stage of discovering neural correlates to relate these findings with phenomenal content of mental states in a meaningful mapping $((\mathrm{Wu}$, 2018)), finally in the peripheries of kernel, the relation is of minimalist type with substrate layers supporting the existence of higher strata e.g. it remains intractable to map the behaviour of brain and neurons to locate the seat of consciousness.

\subsection{Sentient Consciousness Analysis}

Sentient consciousness is one of the higher rank achieved by a conscious being. Humans are an epitome of it, as they manifest numerous advance conscious behaviors like reflective thinking that includes planning, decision making, selecting, making choices and so on. While in this proposal, focus is on de-crypting consciousness at all levels of existences and particular attention is given to sentient being, especially to speculate about the learning mechanism and

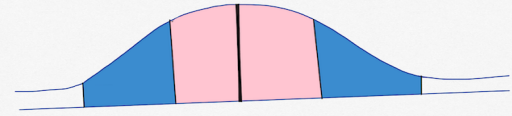

Fig. 3. Kernel relationship between ranks of consciousness.

how structure are formed in minds by learning processes, and also how these structure enable autonomous behaviors? One premise that can explain these autonomous behaviors of animate beings is an effect of an underlying evolving learning structure, to understand apart of this concept, consider an important learning process called habit of mind that may have insight into learning mechanisms of a pattern and behaviors, may it be an action or an abstract concept. This process of mind has an effect of solidifying a learned pattern and plays an important role of easing the cognitive workload and stresses incurred in process of learning a new behavior. After being has practiced same behavior, gradually cognitive load is laid off. All animates including humans act out these learned behaviors seemingly unconsciously or automatically. Here, an extension of being into immediate environment is seen through learning, a kind of adaptation and assimilation mechanism.

Autonomous behaviors through learning reveal the underlying entangled shift in cognition from a conscious to an unconscious state. How such mechanism happen are not completely understood, but both human and their biological cousins demonstrate such learning behaviors and that is a clue of them sharing various mental states and information structures.

In perspective of AGI, It is now known that these autonomous behaviors seen in animate objects are not easily imitable in inanimate machines as no known general formulation of the motivations of a being to act are understood. One more famous caveat of consciousness is "what it is like to be bat" by Nagel (1974), ergo learning structures are only realised by realising a being. Up-to-date any success in simulation of such behaviors in machines are spurious animations. To explain this claim, lets take an example of few autonomous behaviors demonstrated by a robot dog in comparison to the behaviors of real dogs. The former acts, plays and demonstrates similar affinity, body language and emotional responses to that of the behaviors of real dogs. While such manifestations by a robot contraption present a fine simulation of behaviors, they are spurious in the robot dog as they are contrived in well defined programming structure and represent a sense-response model, not like a product of behaviors and being entanglement with environment and no new spontaneous behavior is possible as causes of those actions are unknown to programming and more then that there persists a lack of a connection of self 
with the world map.

Sentient consciousness is an integration of self with the world map, where self assumes world map as its extensions and in this process the eventuating consciousness is not only an information structure rather an organic relation of a self with the world.

\subsection{Thought in Consciousness}

Consciousness if has a basic unit then thought might be one of the most appropriate concept in explicating the function of a consciousness in our vernacular. Thoughts are one possible ways to form the world map, lets speculate, how thoughts are created and effect ideas and concepts. At the start, whenever a new thought is perceived, it may have causes in current perceptions and senses, previous experiences, known facts or there might be unknown and random sources. At early stages of perception, it might have an abstract form and have abstract relations in the mind's world map. The thought reification process is carried out by connecting it to other world map elements, with this developing connections, thoughts are transformed into beliefs. To elaborate this process further, it is supposed that thoughts are grounded gradually in mental representations and seems to take a more elaborated form and structure, like it getting more properties, functions and behaviors, which are associations of thought with a priori elements of world map, a type of rationalising activity on the content of thought. Finally, the thought assumes a more material form and assumes a part in the mind's world map as a type, an item, a concept, an idea or an association.

if there is a needed to develop it further then consciousness building process may take aid by sharing ideas with beings having similar world maps, these processes are of a social nature, viz., discussion, consensus or ideas sharing and group activities. Effectively, all this rigorous casting works on the thought modify it into a more permanent form. For this, sentient beings have empirical and rational tools as mental representation forming mechanism. In this way, new facts accepted by sentient society are discovered and now the thought from an abstract existence has achieved a more validated type, with definite known properties, functions and behaviors. It is not suggested that all of above must happen to a thought in the consciousness but their must be a similar pathway that might effect into new type items, eventually playing a role in extension of the mind's world map.

Thought and thought genesis are basic process of consciousness and both explain elements, associations and structure of mind's world map.

\subsection{Retrospective Analysis}

This retrospective analysis of consciousness is investigated to understand the differences of commitments of theories, ideas and paradigms against the presented paradigm. Our revisit commenced with materialism and the strong assertion of materialism, having commitments in both domains of metaphysics and linguistics, where it explicates whole of reality on premises of the material properties and behaviors, and in the case of consciousness a similar treatise is developed. Though consciousness in common parlance is a niche of higher order brain functions and brain veritably in its very existence is material, therefore it seems natural to attribute consciousness as a sophisticated property of material brain at higher complexity, with integration of brain attributes from physical to functional and consequently to behavioral.

In this proposal, one speculation against strong materialism is in regards to the question of reckoning of a strict causal relationship between wetware and consciousness. This relationship is hard to ascertain from neurons "the unit of physical brain" to mental states and consciousness, and consequence is an unsatisfactory enterprise of a reductionism (Koch (2012)). The basis of this divergence is based on the speculation that reality and consciousness are organised in hierarchy of scales and layers, and relations among layers and scales are functional in nature.

Another popular description of consciousness is based on the metaphysics of panpsychism. In a pure ontological inspection, there is a suggestion of a pro-materialism stance as a strong candidate to explain both: the behaviour of nature and behaviour of consciousness. Similarly, in case of pansychism, it drives strength from pervasive nature of matter with esoteric nature of consciousness to suggest a proto-consciousness. Whereas present paradigm has different commitments from both materialism and pansychism and there is a divergence in classification of consciousness, by appealing to the premise that it is the network of existences in all its behaviors is cause of consciousness and there is no necessity for special allowance at every existence in nature to have consciousness processing units, of the type possessed by sentient beings.

Another aspect of retrospective analysis is the position of presented paradigm. In this context, both views of the Platonist and materialist are considered too dichotomous in comparison to the proposed model of consciousness, and neither pure forms of Plato nor the rigid metaphysics of materialism satisfies the continuum of consciousness. In this conundrum, it seems a reasonable approach to take an exception in interpretation of consciousness, by avoiding strict views of both Plato and materialist. In consequence, the proposed theory of consciousness positions itself in between them.

The premises that allows the claimed divergence is that all properties, behaviors and existences are contingent on scale and layer in reality, and between layers there persists an existence relationship but mapping methods used as in standard approaches to establish relationship between entities in physics is not always possible. To explain this claim further, imagine a painting, the content properties of the painting are fabric, colors and strokes, and the experience subsumes theme, ideas and story of the picture. These properties represent two divergent realities, first group identifies with materialism, while second are experiences in realm of consciousness, which according to proposed paradigm a valuation based on a subjective world map. Both the material and the mental aspects share relationship as far as existence but these relations are not explainable in traditional sense of materialism or panpsychism.

In the last of retrospective analysis, the proposal also intentionally abstain from indulgence in explanation of consciousness as emergence (see history of emergentism by Haugeland (2014). In our view, belief in materialism by 
suggesting everything physical remains an unsatisfactory explanation of reality, and this approach have explanatory gaps, which become more starker in explications of consciousness and so there is an appeal to various metaphysics and one interesting metaphysics is emergentism. Which actually do make good interpretation of enigma of reality and consciousness i.e. from simple units interacting to give rise to complexity. In contrast, the present paradigm is concerned with understanding of consciousness from physical to sentient ranks and the proposed functional nature of relationships among structures in layers of reality is an other potential premise to interpret reality/consciousness and its loose associations.

\subsection{Symbols, Enumerations and AGI}

Symbols are primary raw material of a conscious minds and thoughts represented through symbols are shared among beings to build understanding and knowledge (an intricate model of world). Moreover, symbols are the simple structures that represent relations among existences and a thing means only because it has a relationship with existences around it. Symbols only matter as they are representation of the underlying semantics, and both symbols and semantics share a sophisticated relationship in this paradigm. Semantics on their end are acts of consciousness and symbols play a representative role, where primary entity is consciousness that plays a functional role in accessing and processing of symbols under particular semantics. This structure of symbols under semantics give a qualifiable sense of the world. Like symbols, enumeration of things is an an other representation use by consciousness, it helps in making sense of world in a quantifiable manner. Both symbols and enumerations are fundamental representations of the process of consciousness and are not only defined by the ends they serve, rather to the contrary they are acts, processes and mechanisms to elaborate process of consciousness.

Symbols are essentially void of semantic content sans consciousness, where as it is act of consciousness that complements a use, a role or an event for them. Discerning symbols as packets of consciousness is frivolous assumption, rather it is act of semantics that precedes symbols in an abstract network of relations of existences in the mind's model of the world.

To understand symbols one essential requirement is frame of references as they are the means to localise the semantics of a symbol. Frame of references are thus an aid in the recognition of symbols and enumerations. These are fundamental cues of consciousness in building mind's model of world (e.g. a world map). To understand any pattern, some fundamental references are required, these are the fundamental constructs that seems to self evidently profess their completeness in consciousness and are assumed a priori or taken as they are available. In this paradigm, frames of references are only true and hold validity as much as proximity of scale is maintained. To a great extend, the frame of reference directly accessible to human experience are stable and evidently, they guaranteed both human survival and prosperity, e.g. Eulicdean geometric structures represent intuitive proximal view of reality as things also do appear straight at close distances, while less intuitive geometries such as projective and non-Euclidean are developed much later.

Frame of references are first act of consciousness; symbols and semantics follows as emergent constructs, building complexity upon complexity in an incremental manner. Frame of references are the corner stone of consciousness, subsequently they engender knowledge, understanding and innovation in sentient beings.

The assumption is that perceive reality is an approximated image and equally, consciousness is a necessary consequence of low bandwidth of relations between mind and existences. Reality approximation is like looking through a glass of a particular magnification to observe an effect, consequently all involved elements are sync in perfect synergy to engender spectacle, it is akin to find the focal length of a lens so an appropriate amount of light will converge on retina of an eye to give the expected spectacle in all its perfections. This simple observation tell us that we discover what we set out to find with presumptions and design appropriate apparatus. Interestingly, experiment settings like sensor range and capacities will determine the structure available to us, contrarily try to find a planets with a microscope or use a telescope to find an atom, all such attempts are out of range or scale. Thus measurement is key to determine a reality, and the compatibility of involved existences is an essential element to determine a possible structure.

\section{Conclusion and Perspectives: Formal- IZATION OF CONSCIOUSNESS TOWARDS AGI}

In this proposal, higher rank of consciousness is considered a product of interactive and existence based relations of a being to the proximal reality, this framework of consciousness engenders a map of the world. Interestingly, this map represents an integrated structure of beings, where consciousness is a subjective matter of one node in the network.

One possible attempt to formalise this interactive and associative framework of consciousness is by inquiring the functional outputs like- how forms, symbols and semantics are realised, for example to create abstract knowledge. A theory about these functional processes of consciousness may elaborate the information aspect of consciousness, and this enterprise may prove a step towards materialization of a human like consciousness or at least helps in coming up with novel methods of information representations with incorporating benevolent attributes of both symbolic (notoriously known as GOFAI (Haugeland (2014))) and subsymbolic artificial intelligence (such deep learning, Bayesian networks and evolutionary algorithms). Conclusively, the aim of this publication is to lay foundations for an information structure that can represent the description of reality in form of interactions of existences, while it is important to highlight that functional essence of consciousness may remain elusive for a little more longer.

On the subject of functional aspect of building an artificial consciousness, an inquiry into functional aspect of consciousness may differ in methodology from present day data driven approaches. In functional aspect of consciousness, main focus is to generalise being to beings relations in order to formalise functional solutions to question of intelligence, like identifying a new form of 
proto-consciousness: the basic act and unit of consciousness.

The next project is to formalise consciousness as a set of universal functions, and the functional units of consciousness will be a priori property of existence. To elaborate this, the formation process of the world map is an awareness extending process not necessarily a conscious synthesizing process although both are entangled, so the distinction is necessary at incipient stages of developing a functional property of consciousness, while after achieving a functional consciousness, the progress and development of consciousness with expanding world map are at liberty to increase in complexity.

The functional consciousness approach is not design as an application specific solution, rather existence and interactions among existences are its core commitments, and there is a clear distinction from present paradigm of artificial intelligence, and this distinction is by way of preferring formulations of consciousness and intelligence by way of learning from examples, whereas present day methods of learning follow learning by examples methods (Beyer (2016)). The proposed AGI paradigm consequences into expansion of awareness with synthesis of an awareness.

\subsection{A note on distinction in role of samples in learning}

Learning by example formulation of consciousness or intelligence is rigid as each sample has a definite contribution to generalise the space of description of a pattern, and around this space of description similarity and dissimilarity are establish to achieve automation. Although this is an intuitive solution to pattern learning, unfortunately it is akin to route learning method that is keen on learning symbols with brute force with disregard to semantics. This aspect is a cardinal caveat of present day AI methodologies, and eventually these methods approach a limit of pattern description effect, which is probable hard to resolve and is a source of a cyclic nature to present AI developments, i.e. after every episode of progress there is stagnation in AI. This rigidity is innate in learning by examples AI methodologies and makes them harder to generalise. After tracing the source of this rigidity in present day AI, the proposed paradigm opts for a different approach to consciousness with focus on learning from examples as an awareness extending mechanisms with simultaneously, a pattern forming process. In this way, it has less to do with number of samples or examples and more with specific new information found in a sample and it can be said that every new sample acts as another episode of awareness. In conclusion, the proposed consciousness paradigm is step towards artificial general intelligence, and this paradigm shift is achieved by an alternate formalization of consciousness as an inquiry into set of functions, describing nature of reality by relations among existences, and it is hoped that this may prove to be a worthy contribution towards the development of conscious machines. The idea is to build consciousness embodying machines that incorporate the phenomenological inspired approach to consciousness by incorporating being to beings relations in the form of a mind's world map. The essence is a functional learning mechanisms that are diametrically opposite to both present day data focused approaches of artificial intelligence and also from notorious GOFAI.
The first step is to recognise the modes of existences and consciousness both as a priori attributes of reality mix.

\section{REFERENCES}

T. Alter and D. Pereboom, "Russellian monism," 2019.

R. Descartes, "Meditations and other metaphysical writings, übersetzt von desmond m. clarke," 2003.

D. Papineau, Thinking about consciousness. Clarendon Press, 2002.

J. Haugeland, "Farewell to gofai?" in Speaking Minds. Princeton University Press, 2014, pp. 101-114.

D. Chalmers, "Panpsychism and panprotopsychism," Consciousness in the physical world: Perspectives on Russellian monism, pp. 246-276, 2015.

M. Minsky, Society of mind. Simon and Schuster, 1988.

D. Lloyd, "Consciousness: A connectionist manifesto," Minds and Machines, vol. 5, no. 2, pp. 161-185, 1995.

J. T. Blackledge, "An introduction to relational frame theory: Basics and applications." The Behavior Analyst Today, vol. 3 , no. 4 , p. $421,2003$.

D. C. Dennett, Consciousness explained. Penguin uk, 1993.

S. M. Weiss and N. Indurkhya, "Rule-based machine learning methods for functional prediction," Journal of Artificial Intelligence Research, vol. 3, pp. 383-403, 1995.

J. R. Searle, S. Willis et al., Consciousness and language. Cambridge University Press, 2002.

M. Heidegger, The basic problems of phenomenology. Indiana University Press, 1988, vol. 478.

B. F. Skinner, "Operant conditioning," The encyclopedia of education, vol. 7, pp. 29-33, 1971.

I. Kant, Critique of practical reason. Hackett Publishing, 2002.

D. Chalmers, "The hard problem of consciousness," The Blackwell companion to consciousness, pp. 225-235, 2007.

D. Beyer, Future of Machine Intelligence. O'Reilly Media, Incorporated, 2016.

P. Ricoeur, Husserl: An analysis of his phenomenology. Northwestern University Press, 1967.

J. Kim, Supervenience. Routledge, 2017.

W. Wu, "The Neuroscience of Consciousness," in The Stanford Encyclopedia of Philosophy, Winter 2018 ed., E. N. Zalta, Ed. Metaphysics Research Lab, Stanford University, 2018.

T. Nagel, "What is it like to be a bat," Readings in philosophy of psychology, vol. 1, pp. 159-168, 1974.

C. Koch, Consciousness: Confessions of a romantic reductionist. MIT press, 2012.

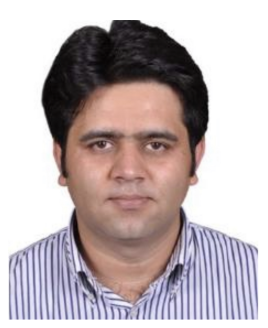

Adnan Rashid Chaudhry received his BS. software engineering in 2005 from NUML, Pakistan, Masters in computer engineering in 2006 from INSA, Lyon, France, and PhD computer engineering in 2009 from Ecole des Mines de Paris, France. He has worked as a postdoctoral researcher at ImageLab, UNIMORE, Modena, Italy (2010-2012) and in dept. of electrical engineering, University of lowa, USA (2012-2014).

Presently, teaches as an asst. professor in dept. of Computer Science, FCIT, NBU, KSA His engagements are developing models, theories and frameworks of Artificial Consciousness and Intelligence. 